\title{
Bionics: an introduction
}

\section{Editorial}

Given an opportunity to write an editorial opinion for this esteemed journal, I acknowledge the contribution of the entire author's, reviewers, editors and other team members of the journal. I am thankful to all contributors for submitting their valuable research works. Knowing one's own interest is not an easy goal to achieve with perfection though. However, a researcher in the domain of Bionics and Biomechanics should develop intense interest in studying the impact and applications of engineering, science and technology in research, design, development and challenges in the field of bionics and Biomechanics. The "Journal of Applied Bionics and Biomechanics" is a very good platform for disseminating research work, ideas and innovations at internationally recognized platform. Bionics is the study of the mechanical systems that functions like living matter or the part of the living matter. The field of Bionics is therefore very different from bioengineering or biotechnology, in which the various tasks are performed by applying the concepts of nature for example digesting of wastes by bacteria in biochemical batteries to supply electrical energy. Since long human beings are getting inspired from the nature and trying to implement the concepts inspired from animals or other living organisms. This mimicking of nature has its own inherent advantages as the design of nature in doing certain tasks is perfect and if the machines are designed on the basis of these concepts, these can really be excellent in performing the assigned task. Although exact imitation of the natural system is difficult to implement, due to various factors like change in environment, size, difference in scale and many other factors which have direct influence on the design. Therefore, instead of unswervingly imitating the design bionics researcher dwells on understanding the underlying concept of how the things are working in natural environment. The study of Living beings is done from several points of view to get inspiration from nature. Some examples of such cases are:

a. Muscles of a living organism are more efficient than mechanical motors

b. Efficiency of storing solar energy by plants is many times more than the Solar cells

c. Latest telephone exchanges cannot compete with the swiftness of transmission of information within the nervous system.

d. Even the most powerful supercomputers cannot match the capacities of Human brain.

From the above examples fields of bionics research can be categorized in to two main domains of:

\section{i. Information Processing \\ ii. Energy Transformation and Storage.}

The general pattern of the information network of living organisms is the following: environmental sensations are received by the organs of sense and then coded into signals that are transmitted by nerves to the centers of processing and memorization of the brain. Ant colony has cooperative system also known as swarm intelligence. The ants are so sensitive that they can detect a obstruction in their path a few meters' distance through collaborative communication. Though
Volume I Issue 3 - 2017

\author{
Kshitij Shinghal \\ Department of Electronics \& Communication engineering, \\ Moradabad Institute of Technology, India
}

Correspondence: Kshitij Shinghal, Department of Electronics \& Communication engineering, Moradabad Institute of Technology, India, Email kshinghal@gmail.com

Received: October 10, 2017 | Published: October II, 2017

much more sensitive man-made infrared detectors exist, bionics can still profit from study of the ants for robotics and other applications. First, it would be interesting and of potential value to understand the principle of energy transformation occurring hen a frog is under hibernation, as well as the process by which the nerves are stimulated in the absence of any stimulating mechanism. Another striking example is the color changing organ of the chameleon. If we take the example of an optical fiber, the signal is attenuated as it travels along the fiber, and the signal has to be amplified by putting amplifiers in between. This is not the case for the animal nerve axon; the neural impulse issued from sense organs does not weaken in travelling along the axon. This impulse can travel in only one direction. These properties make the nerve axon capable of logic operations. Recently a semiconductor device called a neuristor was designed based on the properties of nerve axon. This device neuristor was capable of propagating a signal in one direction without attenuation and able to perform numerical and logical operations. These are only a few examples of the vast possibilities of developing and improving the things based on the concepts derived from nature however the possibilities and the scope are limitless. As a conclusion, we can say that bionics can be used in various applications as simple as a wooden leg and joint, to a brain operated leg that connects to your nerves and moves more naturally than other prosthetic. More and more innovative ideas are always being brought up and we continue to advance in our quest to copy the living organisms and nature as best we can.

MedCrave Online Journal of Applied Bionics and Biomechanics (MOJABB) is an International, Peer reviewed Journal of advanced technological developments based on the science of biological systems. Please contribute articles to this journal in a timely manner, to ensure it gets published timely and becomes part of this important forum for the exchange of ideas and knowledge which will ultimately translate to better technological exchange of information among peers. The journal covers all the aspects of bionic science and engineering including fundamental understandings of animals and plants for bionic engineering, such as locomotion and behaviors of animals, structures, composites, morphology and physical properties of plants and natural materials, applications of such understandings in engineering, technology and designs. The Journal accepts Research Papers, Review Articles, Short Communications, Case Reports, Mini-Reviews, Opinions, Letter to Editors, etc. in this field which will be enlightening the scientific community. 


\section{References and acknowledgement}

I have based this paper on the materials collected from several courses I've attended. It is inspired by many Guidelines for Authors of various reputed international journals. Some of this information is also featured in various tutorials available online. In addition, I have consulted several web pages, blogs etc. for shaping this article. I would like to thank Mr. Amit Saxena and Ms. Deepti Shinghal for their valuable support, without their help this article would have been impossible to complete.
Dr. Kshitij Shinghal is currently working as an Associate Professor in MIT Moradabad with 18 years of experience in teaching and research. His research areas of interest include Embedded Systems, Biomedical and Bioelectronics, VLSI Design, Wireless Sensor Networks, and Ad-Hoc Networks. He has published more than 60 research articles in International Journals and Conference Proceedings. He is currently a lifetime member of 07 International Societies.

\section{Conflict of interest}

Author declares that there is no conflict of interest. 